Asian J Agric \& Biol. 2021(1).

\title{
A wheat stress induced WRKY transcription factor TaWRKY32 confers drought stress tolerance in Oryza sativa
}

\begin{abstract}
Essam Darwish 1,2 , Shoaib Ur Rehman 2,3, Xinguo Mao2*, Ruilian Jing²
${ }^{1}$ Plant Physiology Section, Agricultural Botany Department, Faculty of Agriculture, Cairo University, 12613 El Gamma Street, Giza, Egypt

${ }^{2}$ National Key Facility for Crop Gene Resources and Genetic Improvement, Institute of Crop Sciences, Chinese Academy of Agricultural Sciences, Beijing 100081, China

${ }^{3}$ Institute of Plant Breeding and Biotechnology, Muhammad Nawaz Shareef University of Agriculture, Multan, Pakistan

\section{Abstract}

WRKY transcription factors group play a significant role in stress signalling pathway. In this study, a WRKY member of bread wheat, TaWRKY, was cloned and its function in response to water stress conditions was studied. Expression analysis revealed that TaWRKY32 mainly expressed when plants were subjected to PEG, $\mathrm{NaCl}$, and cold and probably participate in ABA dependent signalling pathways. This study was done to elucidate the role in transgenic breeding, TaWRK32 was transferred to rice plants under CaMV-35S promoter. Overexpression of TaWRK32 lead to enhanced fresh shoot and root weight at seedling stage when compared to wild type (WT). Moreover, transgenic rice lines under water stress, showed greater recovery rate when compared to WT. Higher values of cell membrane stability and lower $C h l a / b$ ratio of transgenic rice lines under water stress conditions markedly indicated the importance of TaWRKY32 under water stress conditions. These results suggested that TaWRK32 has multifunctional and might be an instrumental tool in breeding for transgenic plants for improved water stress tolerance in wheat.
\end{abstract}

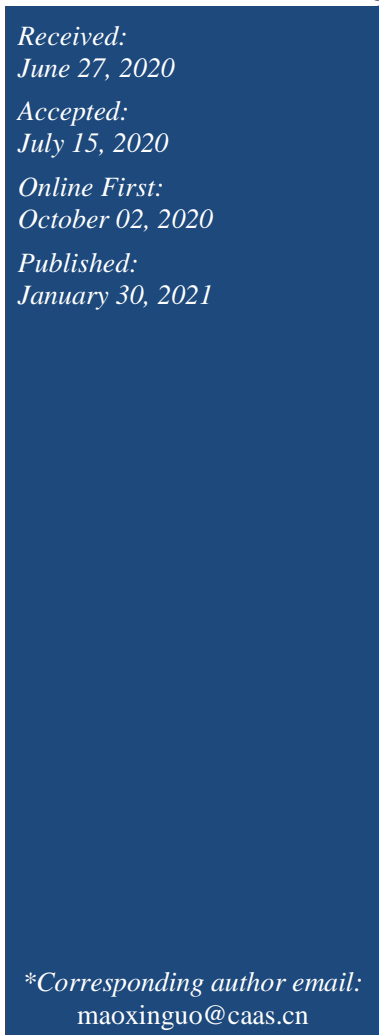

Keywords: WRKY transcription factor, Drought stress, Cell membrane stability, Chlorophyll contents

\section{How to cite this:}

Darwish E, Rehman SU, Mao X and Jing R, 2021. A wheat stress induced WRKY transcription factor TaWRKY32 confers drought stress tolerance in Oryza sativa. Asian J. Agric. Biol. 2021(1). DOI: https://doi.org/10.35495/ajab.2020.06.371

This is an Open Access article distributed under the terms of the Creative Commons Attribution 3.0 License. (https://creativecommons.org/licenses/by/3.0), which permits unrestricted use, distribution, and reproduction in any medium, provided the original work is properly cited.

\section{Introduction}

Plants are effected by multiple environmental stresses i.e., salt, drought, and high temperature that lead towards yield decline (Nakashima et al., 2009). Crop plants have developed distinctive strategies via physical adaptation, molecular and cellular changes to adapt against multiple abiotic stresses (Knight and Knight, 2001). Regulatory mechanisms in plants are mainly mediated by transcription factors (TFs) that govern the expression of genes when subjected to environmental and developmental changes (Buscaill and Rivas, 2014). During abiotic stress, the activity regulations of TFs lead to alteration in the gene of interest. TFs such as AP2/EREBP (Xu et al., 2011), MYC/MYB (Abe et al., 1997), HSE binding (Fiorenza et al., 1995), NAC (Nakashima et al., 1995), and WRKY TFs (Eulgem et al., 2000) generally contribute 
to plant adaptation to abiotic stresses. Among the TFs, WRKY are mainly present in plants e.g. Oryza sativa, Arabidopsis thaliana, Setaria italica, Glycine max and Triticum aestivum, which confirm the role of WRKY TFs in plant response to stress tolerance (Chen et al., 2017).

Plant WRKY TFs play important roles against biotic stress factors, sugar signalling, senescence, root growth and hormone signalling (Bakshi and Oelmuller, 2014). For instance, 61 of the PtrWRKY genes are over-expressed by both biotic and abiotic stressors in Populus (Jiang et al., 2014). Furthermore, TaWRKY1 facilitates stomatal movement through an ABA-dependent pathway (Ding et al., 2016), while TaWRKY10 has a significant role under cold, drought, salinity, and enhance tolerance of transgenic tobacco lines to stress conditions (Wang et al., 2013). Among abiotic stresses, elevated salt stress highly hinders plant growth, while at different plant growth stages the higher salinity caused increased reactive oxygen species (ROS), metabolic toxicity, reduce nutrient acquisition and inhibition of photosynthesis and metabolism (Hasegawa et al., 2000).

Wheat plant is an important crop and has an economic significance to the world, while its production is reduced due to environmental stresses, such as; water stress, salinity and high temperatures (Hu et al., 2012). Therefore, the current study was designed to (i) study the expression level of TaWRKY32 gene at seedling stage in response to different abiotic conditions (ii) study the morphological characteristics of TaWRKY32-overexpressing rice lines under control and water stress conditions.

\section{Material and Methods}

\section{Plant material, growth conditions and stress treatment}

Drought tolerant wheat genotype "Hanxuan 10" was used in this study. Seeds were subjected to tap water in a growth chamber $\left(20 \pm 1^{\circ} \mathrm{C}\right.$ and $150 \mu \mathrm{mol} \mathrm{m}^{-2} \mathrm{~s}^{-1} 12$ $\mathrm{h}$ light/12 $\mathrm{h}$ dark cycle). Wheat seedlings, at two-leaf stage, were treated with $250 \mathrm{mM} \mathrm{NaCl}$, PEG-6000 ($0.5 \mathrm{MPa})$ solution, $50 \mu \mathrm{M} \mathrm{ABA}$ or cold $\left(4^{\circ} \mathrm{C}\right)$ as described by Mao et al. (2010). Roots samples were collected at $0,0.5,1,1.5,2,3,6,12,24,48$, and $72 \mathrm{~h}$ after exposing wheat seedlings to aforementioned abiotic stress factors followed by storage in liquid nitrogen and stored at $-80^{\circ} \mathrm{C}$ for RNA extraction.

Wild type (WT) as well as transgenic rice were grown at the Experimental Station of the Institute of Crop
Sciences, Chinese Academy of Agricultural Sciences, Beijing, China in an area confined for transgenic plant cultivation in $2017\left(39^{\circ} 48^{\prime} \mathrm{N}, 116^{\circ} 28^{\prime} \mathrm{E}\right)$. Morphophysiological assays were done on $\mathrm{T}_{3}$ homozygous transgenic rice lines expressing TaWRKY3. For plant height (PH), tillers per plant (TPP) and spike length (SPL), transgenic rice material was planted following general agronomic practices having $25 \mathrm{~cm}$ row to row distance. Transgenic rice and WT were also planted in small plastic container (length $\times$ width $\times$ height $=28$ $\times 20 \times 9 \mathrm{~cm})$ for 4 weeks following randomized complete block design (RCBD) in duplication. Ten 4 week-old seedlings were selected to measure seedling traits such as root fresh weight, shoot fresh weight and root/shoot ratio $(\mathrm{R} / \mathrm{S})$.

\section{Cloning TaWRKY32 and sequence analysis}

Full-length cDNA sequence of the TaWRKY32 gene was got by sequencing a wheat cDNA library. To generate transgenic rice, the ORF of TaWRKY32 was amplified with primers (F: ctggtaccATGGCACAGCCTTCTCC; R: ctgtcgacCTATTATTTACATCTGGGGACC) by TransStart fast $P f u$ DNA polymerase, and inserted into binary vector pCAM1390 with modification after digestion with KpnI and SalI. The construct was then transferred into rice by Agrobacterium mediated method as followed by Mao et al. (2010).

\section{Expression patterns of TaWRKY32 in wheat} RNA was extracted with RNAprep pure Plant Kit (TIANGEN, Beijing) and cDNA was synthesized with a SuperScript ${ }^{\circledR}$ Double-Stranded cDNA Synthesis Kit (Invitrogen). Quantitative Real-time PCR (qRT-PCR) was done in three replicates with a Roche LightCycler 96 Real-Time PCR system (Roche, Switzerland) using the SYBR Green PCR Master Mix Kit (TaKaRa, Japan). Wheat Actin gene and rice Tubulin gene were used as house-keeping genes to detect the relative transcript level of TaWRKY32 in wheat and rice. PCR conditions were pre-incubated at $95^{\circ} \mathrm{C}$ for $2.5 \mathrm{~min}$, followed by $95^{\circ} \mathrm{C}$ for $10 \mathrm{~s}, 60^{\circ} \mathrm{C}$ for $20 \mathrm{~s}$, and $72^{\circ} \mathrm{C}$ for $20 \mathrm{~s}$ for 40 cycles. For each gene, the relative transcript level was measured using the $2^{-\Delta \Delta C \mathrm{~T}}$ method as followed by Mao et al. (2010). $\Delta \Delta C_{\mathrm{T}}=\left(C_{\mathrm{T}}\right.$, interested $-C_{\mathrm{T}}$, internal control $)$ Time $_{\mathrm{x}}-\left(C_{\mathrm{T}}\right.$, interested $-C_{\mathrm{T}}$, internal control $)$ Time $_{0} .\left(C_{\mathrm{T}}\right.$, cycle threshold; $C_{\mathrm{T}}$, interested, $C_{\mathrm{T}}$ of the interested gene; $C_{\mathrm{T}}$, internal control, $C_{\mathrm{T}}$ of the control gene). Time ${ }_{\mathrm{x}}$ represents the time of sample collection while Time ${ }_{0}$ represents the time prior to treatment. TaActin (F- 5'- 
CTCCCTCACAACAACAACCGC-3'; R- 5'TACCAGGAACTTCCATACCAAC-3') was used as an internal control gene. To detect transcript level of TaWRKY32, The primers (F- 5' ATCCACAGTTATTCGATCACCCAT 3'; R - 5', CATTGTTGAGTTAGCCTCTGAAGC $3^{\prime}$ ) were used for TaWRKY32.

\section{Drought tolerance assay}

Drought tolerance assay was done at seedling stage. $\mathrm{T}_{3}$ generation of transgenic rice lines with relatively higher expression level were selected for phenotyping under drought stress conditions. Transgenic rice seeds were germinated by soaking them in water for three days. After that, Seedlings were transplanted in plastic container (length $\times$ width $\times$ height $=60 \times 42 \times 12 \mathrm{~cm}$ ). Transgenic rice lines and WT were grown in container following RCBD with duplication. Watering of Two weeks old seedlings was stopped at the $3^{\text {rd }}$ week for one week followed by re-irrigation.

\section{Cell membrane stability and chlorophyll contents assay}

Cell membrane stability (CMS) was measured using a conductivity meter (DDS-1, YSI). Leaf sample of 3 $\mathrm{cm}^{2}$ from each line was taken in two replications. The harvested seedlings were rinsed thoroughly and immersed in $20 \mathrm{~mL} \mathrm{ddH}_{2} \mathrm{O}$ at room temperature. CMS was measured in terms of percentage as $\mathrm{CMS} \%=$ $(\mathrm{C} 2-\mathrm{C} 1) / \mathrm{C} 2 \times 100$, where $\mathrm{C} 1$ and $\mathrm{C} 2$ are the initial and final conductance, respectively as described by Rehman et al. (2016)

Chlorophyll $a(C h l a)$ and chlorophyll $b(C h l b)$ was determined by using $\mathrm{N}$, N-di-methyl formamide (DMF) as described by Sibley et al. (1996). Leaves samples $(\sim 1 \mathrm{~g})$ were immersed in DMF for overnight at $4{ }^{\circ} \mathrm{C}$ (in duplication). Chlorophyll contents were quantified using spectrophotometer. The extinction coefficient of $C h l a$ and $C h l b$ for high extinction wavelength were $663 \mathrm{~nm}$ and $645 \mathrm{~nm}$, respectively. $C h l a$ and $C h l b$ were calculated by using formula $C h l a=12.10 \mathrm{~A}_{663}-2.74 \mathrm{~A}_{645}, \quad C h l \quad b$ $=21.94 \mathrm{~A}_{645}-5.06 \mathrm{~A}_{663}$ as followed by Rehman et al. (2016)

\section{Statistical analysis}

Estimates of variance was calculated using Microsoft Excel 2013. Results were considered statistically significant at $P<0.05$.

\section{Results}

Expression levels of TaWRKY32 in response to different abiotic stress

The expression pattern of TaWRKY32 was identified by qRT-PCR. Analysis exhibited that TaWRKY32 transcript in roots of wheat seedlings showed different response when subjected to different abiotic stress conditions of PEG, NaCl, ABA and cold (Fig. 1). The expression pattern and highest expression levels varied remarkably for each kind of abiotic stress. In response to $\mathrm{NaCl}$ stress, TaWRKY32 expression did not exhibited any response at early time points of the stress but it reached to its peak at $6 \mathrm{~h}$ and remained highly expressive till $24^{\text {th }} \mathrm{h}$. At early time points, the expression levels have no obvious changes in response to ABA treatment. ABA treatment led to strong upregulation at $72 \mathrm{~h}$. The early strong accumulation of TaWRKY32 transcript was noticed during cold treatment; the transcript was gradually peaked after 1 $\mathrm{h}$ then by gradual decrease in the later time points. When plants were subjected to PEG, an early distinct increase in transcription was found, after $0.5 \mathrm{~h}$. Then, the transcript remains on peak for $2.5 \mathrm{~h}$ and dropped after $3 \mathrm{~h}$. The transcript peaked again after $6 \mathrm{~h}$ followed by decrease to control level after $12 \mathrm{~h}$. These results suggested that the expression levels of TaWRKY32 were enhanced by ABA, drought, ABA and cold treatment.
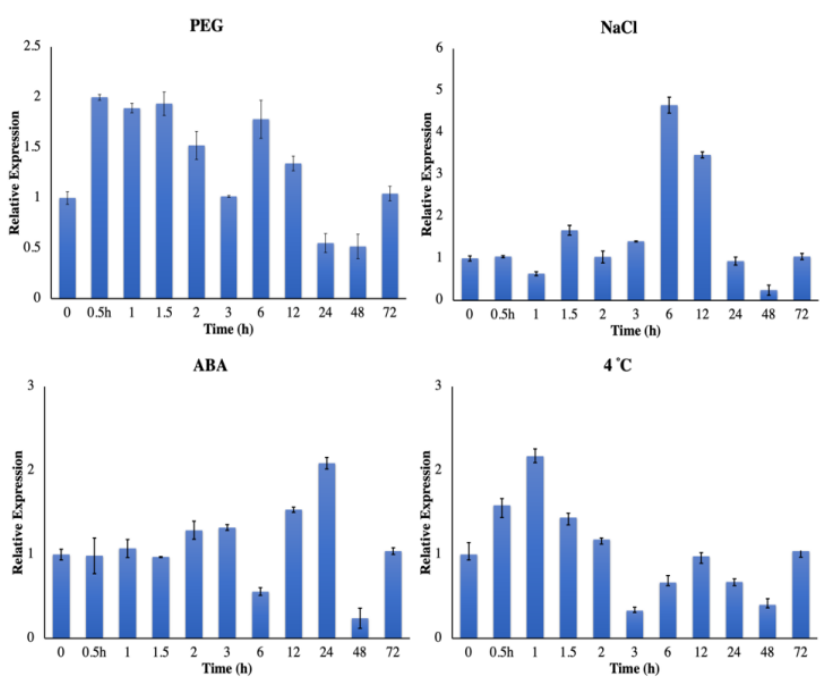

Figure-1. Expression levels of TaWRKY32 in roots in response to different environmental stresses TaWRKY32 effects on transgenic rice growth parameters under control conditions 
To study the effect of TaWRKY32 in transgenic rice lines, the phenotypes of transgenic rice were investigated at different stages of plant development (Fig. 2-4). After 4 weeks, plant height, fresh shoot weight, root length and fresh root weight were measured. At heading stage, $\mathrm{PH}$ showed significant reduction in all transgenic rice lines when we compared them to WT. Meanwhile, TPP did not show significant difference among transgenic rice lines and WT. For SPL, there was significant reduction in L2, L3 and L5 when compared to WT (Fig. 2).
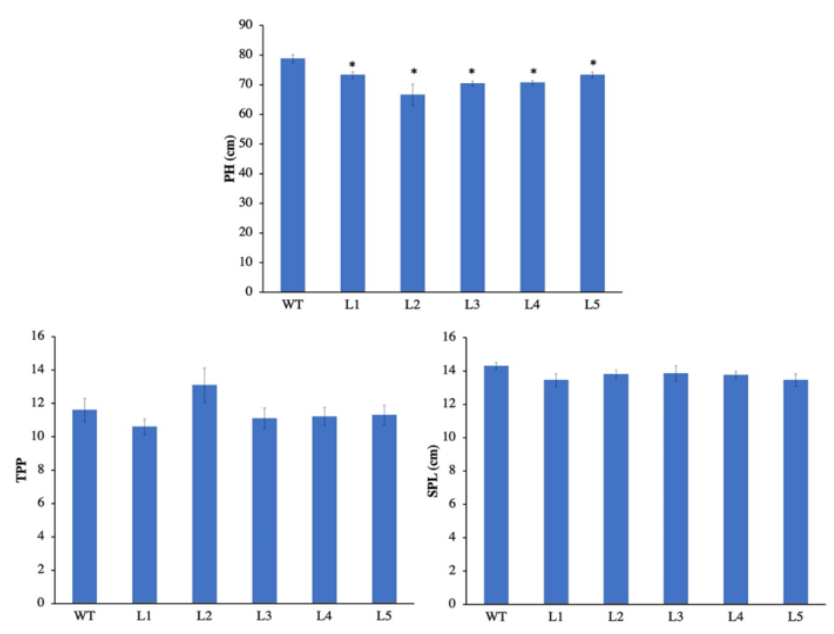

Figure-2. Growth parameters of TaWRKY32 overexpressing rice lines cultured in normal conditions at heading stage. Plant height $(\mathbf{P H})$, Tillers per Plant (TPP), Spike Length (SPL). $*=P$ values less than 0.05 .
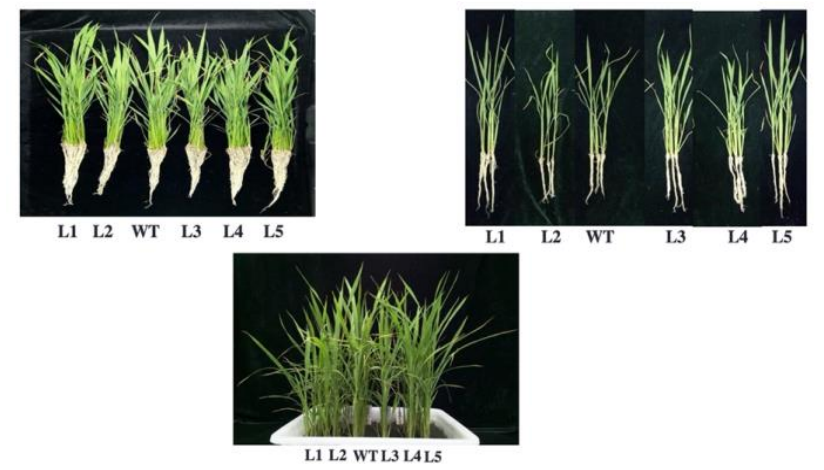

Figure-3. TaWRKY32 - overexpressing rice lines after four weeks from culturing in normal condition.
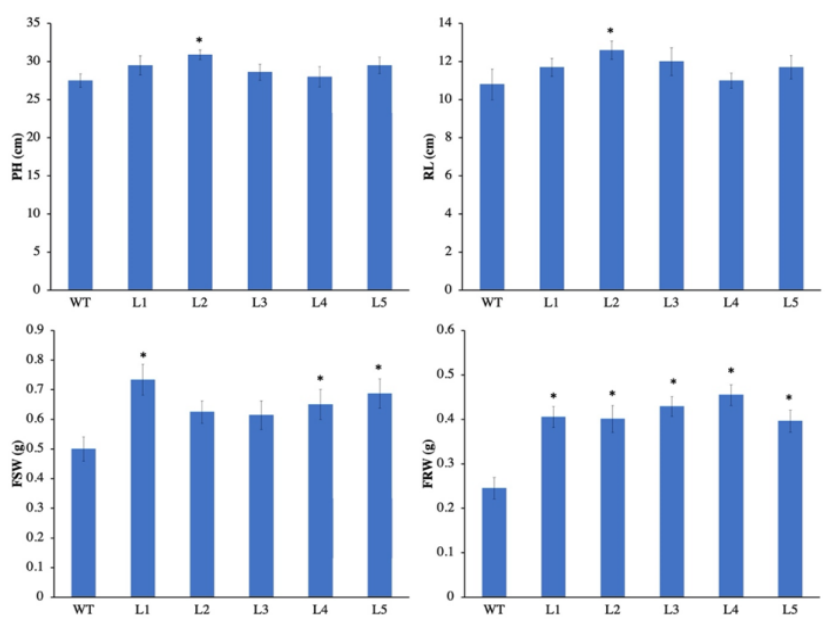

Figure-4. Growth parameters of TaWRKY32 overexpressing rice lines cultured in normal conditions after four weeks. Plant height (PH), Root Length (RL), Fresh Shoot Weight (FSW), Fresh Root Weight $($ FRW). $*=P$ values less than 0.05

In regards to 4 week old plants, L1 and L2 showed significant increment in plant height $(\mathrm{PH})$ and root length (RL) in comparison to WT. All transgenic rice lines showed significant increment in fresh weight of shoot and root in comparison to WT (Fig. 3 and 4).

\section{Drought resistance indexes in the transgenic rice}

We applied drought stress treatment on 2-week-old plants of $\mathrm{T}_{3}$ generation of transgenic rice lines showing relatively higher expression level of TaWRKY32. After withholding irrigation for one week, WT as well as all transgenic rice lines becomes wilted. The plants were re-watered for one week. After recovery from drought, some transgenic rice lines showed more recovery with green leaves in comparison to WT (Fig. 5). To discover the physiological alterations in TaWRKY32 transgenic rice, we applied physiological assays for both WT and transgenic rice. CMS and $C h l a / b$ were determined (Fig. 6). The results showed that all transgenic rice lines except L5 showed slight increase in CMS when compared to WT. Also $C h l a / b$ is used as a valuable marker to assess drought stress tolerance. Our experiment showed that $C h l a / b$ ratio was less in transgenic rice lines than that found in WT. L1 and L3 showed $C h l a / b$ of $70 \%$ and $28 \%$ reduction, respectively. 

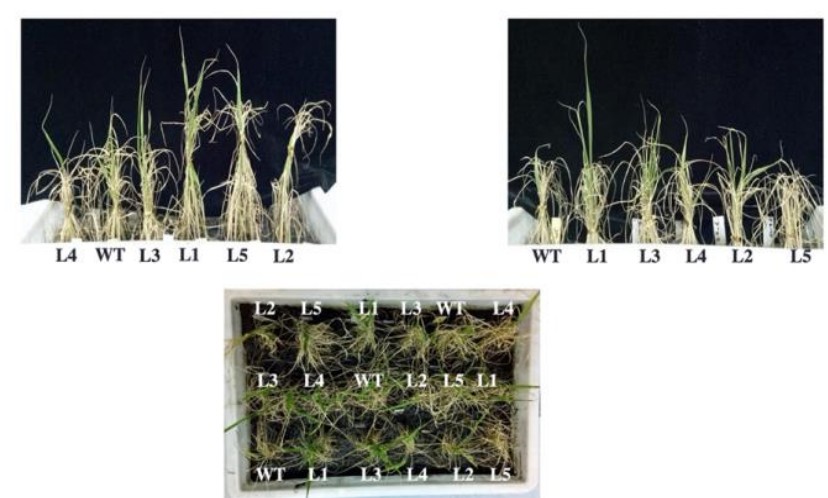

Figure-5. Recovery of TaWRKY32 - overexpressing rice lines after drought stress. Four-weeks old seedlings were subjected to stop watering for one week followed by re-irrigation for one week.
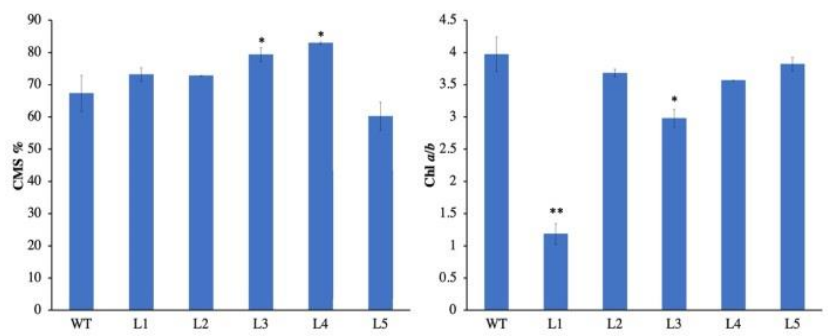

Figure-6. Cell Membrane Stability (CMS) and chlorophyll a / chlorophyll b $(\mathrm{Chl} \mathrm{a} / \mathrm{b})$ ratio of two week-old seedlings of WT vs TaWRKY32 overexpressing rice lines after drought stress recovery. $*, * *=P$ value less than 0.05 and 0.01 , respectively

\section{Discussion}

TFs in plants are considered important player against abiotic stress. Multiple studies showed that overexpression of TFs lead to increase tolerance of plants against environmental stresses. WRKY family is among the most key transcription factors in plants and their role in environmental stress tolerance has been demonstrated in many studies. They form significant parts of signalling pathways governing different plant metabolic processes (Rushton, et al., 2010).

In our study we found that TaWRKY32 expression were induced by different abiotic stresses, for example, $\mathrm{NaCl}, \mathrm{PEG}$, cold and $\mathrm{ABA}$. The trend and the timing of response varied from stress to another. TaWRKY32 transcripts in roots were rapidly accumulated under PEG and cold within 0 to $0.5 \mathrm{~h}$ after treatment. Meanwhile, transcript of this gene reach its peak late after $6 \mathrm{~h}$ and $24 \mathrm{~h}$ when plants were treated with $\mathrm{NaCl}$. The early time points did not show clear induction of TaWRKY32. The same trend was found when plants were treated with ABA. The expression was strongly induced after $48 \mathrm{~h}$ and $72 \mathrm{~h}$. The expression in the early time points has no changes compared to control, with slightly increase after $3 \mathrm{~h}$. These results explain how variable the response of TaWRKY32 under different abiotic stress conditions. Qin et al. (2015) analysed transcription of TaWRKY39, and reported that the transcripts of TaWRKY39 quickly upregulated by $\mathrm{NaCl}$ and $\mathrm{ABA}$ after $0.5 \mathrm{~h}$ from the treatment.

In plants more than 160 WRKY TFs have been classified according to their sequence alignment, motif type and phylogenetic relationship analysis as reported by Okay et al., (2014) which can help to study the stress-responsive genes in wheat (Ning et al., 2017). Many investigations showed that the expression of $W R K Y$ genes fluctuated by hormonal treatment and environmental stresses. TaWRKY genes have several cis-acting elements linked to environmental stresses, elucidating the roles of WRKY genes participating against environmental stresses in wheat (Ning et al., 2017). In general, our results showed that TaWRKY32 is involved in different abiotic stress responses in agreement with previous studies (Jiang et al., 2014).

Abrupt increase in the transcript levels of TaWRKY32 under PEG and cold stress imply its importance at the first stages of stress response, which was in agreement with Zhou et al., 2019 who found that the peak of TaWRKY13 transcript was after $1 \mathrm{~h}$ of treatments. The late response of TaWRKY32 when subjected to $\mathrm{NaCl}$ treatment may indicate that such transcription factor is a key player in different environmental stress conditions, and its expression is regulated according to the stress condition. This late response of TaWRK32 transcript, $6 \mathrm{~h}$ after $\mathrm{NaCl}$ treatment found in our study was consistent with the response of other TaWRKY genes which shows delay in their response to $\mathrm{NaCl}$ (Zhou et al., 2019). This implies that there is difference in how fast these TaWRKY genes respond to different stresses which may explained by the different roles of such genes in abiotic stress.

When a cell is affected by drought stress, plasma membranes are one of the first components that undergoes changes and are affected, and membrane integrity under such stressed conditions is one of the central indicator of water stress tolerance in plants (Blum and Ebercon, 1981). Chlorophyll contents and CMS are counted as valuable biochemical parameters for water stress tolerance (Blum et al., 2001). In our 
results, transgenic rice lines, except L2, showed higher CMS in comparison to WT.

In plants, Changes in chlorophyll contents occurred when they encountered drought stress and that may be correlated with accelerated degradation of the chlorophyll or with biosynthesis defect. The photosynthetic activity in plants under stressful conditions may be reduced. This reduction of photosynthetic activity may arise from the reduction of chlorophyll content. However, in our experiments, the ratio of $C h l a / b$ was less in transgenic lines of rice plants than found in wild type, suggesting that the transgenic rice lines may be able to tolerate water stress. The membrane stability was found to be decreased when plants were subjected to water stress in wheat cultivars (Simova-Stoilova et al., 2008). So, plants have a lower $C h l a / b$ ratio show a greater tolerance level and adaptability to environmental stress conditions. In wheat plants, cultivars with higher CMS percentages and chlorophyll contents showed higher yield (Blum et al., 2001; Khakwani et al., 2012). In regard to CMS in our experiment, L4 transgenic line recorded $33 \%$ increase followed by L5, L3 and L1 which recorded $30 \%, 26 \%$, and $26 \%$ over WT, respectively. These results may indicate the transgenic rice lines with more CMS and less $C h l a / b$ ratio has a potential for drought tolerance and high yield. These results agree with work from Rehman et al. (2016) who investigated the CMS and chlorophyll content in 50 wheat cultivars and found differences among them. CMS and $C h l a / b$ ratio are valuable physiological parameters to be considered as they are positively correlated with plant yield.

These results showed preliminary importance of TaWRKY32 in drought tolerance. For better understanding the role of TaWRKY 32 in plants, more research should be done.

\section{Conclusion}

The study highlighted the importance of the TaWRKY32 TF, in transgenic rice lines in response to water stress. TaWRKY32 expression was found to fluctuate with time when plants were subjected to multiple environmental stresses. $T_{3}$ homozygous transgenic rice lines were evaluated under drought stress, which showed tolerance to water stress as reflected by their potential to recover after withholding water. Beside this, more CMS and less $C h l a / b$ ratio were found as, key indicators for drought tolerance.

\section{Acknowledgement}

We gratefully thank the Talented Young Scientist Program (Egypt-16-040), Ministry of Science and Technology (MOST) of China for supporting this work.

Disclaimer: None.

Conflict of Interest: None.

Source of Funding: This study was funded through grant of Talented Young Scientist Program (Egypt-16040), Ministry of Science and Technology (MOST) of China.

\section{References}

Abe H, Yamaguchi-Shinozaki K, Urao T, Iwasaki T, Hosokawa D and Shinozaki K, 1997. Role of Arabidopsis MYC and MYB homologs in droughtand abscisic acid-regulated gene expression. The Plant Cell. 9(10): 1859-1868.

Bakshi M and Oelmuller R, 2014. WRKY transcription factors: Jack of many trades in plants. Plant Singal. Behav. 9(2): e27700.

Blum A and Ebercon A, 1981. Cell membrane stability as a measure of drought and heat tolerance in wheat 1. Crop Sci. 21(1): 43-47.

Blum A, Klueva N and Nguyen HT, 2001. Wheat cellular thermotolerance is related to yield under heat stress. Euphytica. 117(2): 117-123.

Buscaill P and Rivas S, 2014. Transcriptional control of plant defence responses. Curr. Opin. Plant Biol. 1(20): 35-46.

Chen J, Nolan TM, Ye H, Zhang M, Tong H, Xin P, Chu J, Chu C, Li Z and Yin Y, 2017. Arabidopsis WRKY46, WRKY54, and WRKY70 transcription factors are involved in brassinosteroid-regulated plant growth and drought responses. The Plant Cell. 29(6) : 1425-1439.

Ding W, Fang W, Shi S, Zhao Y, Li X and Xiao K, 2016. Wheat WRKY type transcription factor gene TaWRKYl is essential in mediating drought tolerance associated with an ABA-dependent pathway. Plant Mol. Biol. Rep. 34(6): 1111-1126.

Eulgem T, Rushton PJ, Robatzek S and Somssich IE, 2000. The WRKY superfamily of plant transcription factors. Trends Plant Sci. 5(5): 199206. 
Fiorenza MT, Farkas T, Dissing M, Kolding D and Zimarino V, 1995. Complex expression of murine heat shock transcription factors. Nucleic Acids Res. 23(3): 467-474.

Hasegawa PM, Bressan RA, Zhu JK and Bohnert HJ, 2000. Plant cellular and molecular responses to high salinity. Annu. Rev. Plant Biol. 51(1): 463499.

Hu W, Yuan Q, Wang Y, Cai R, Deng X, Wang J, Zhou S, Chen M, Chen L, Huang C and Ma Z, 2012. Overexpression of a wheat aquaporin gene, TaAQP8, enhances salt stress tolerance in transgenic tobacco. Plant Cell Physiol. 53(12): 2127-2141.

Jiang Y, Duan Y, Yin J, Ye S, Zhu J, Zhang F, Lu W, Fan D and Luo K, 2014. Genome-wide identification and characterization of the populus WRKY transcription factor family and analysis of their expression in response to biotic and abiotic stresses. J. Exp. Bot. 65(22): 6629-6644.

Khakwani AA, Dennett MD, Munir M and Baloch MS, 2012. Wheat yield response to physiological limitations under water stress condition. J. Anim. Plant Sci. 22(3): 773-780.

Knight $\mathrm{H}$ and Knight MR, 2001. Abiotic stress signalling pathways: specificity and cross-talk. Trends Plant Sci. 6(6): 262-267.

Mao X, Zhang H, Tian S, Chang X and Jing R, 2010. TaSnRK2.4, an SNF1-type serine/threonine protein kinase of wheat (Triticum aestivum L.), confers enhanced multistress tolerance in Arabidopsis. J. Exp. Bot. 61: 683-696.

Nakashima K, Takasaki H, Mizoi J, Shinozaki K and Yamaguchi-Shinozaki K, 1995. NAC transcription factors in plant abiotic stress responses. BBA. Gene Regul. Mechan. 1819(2): 97-103.

Nakashima K, Ito Y and Yamaguchi-Shinozaki K, 2009. Transcriptional regulatory networks in response to abiotic stresses in Arabidopsis and grasses. Plant Physiol. 149(1): 88-95.

Ning P, Liu C, Kang J and Lv J, 2017. Genome-wide analysis of WRKY transcription factors in wheat (Triticum aestivum L.) and differential expression under water deficit condition. Peer J. 4(5): e3232.

Okay S, Derelli E and Unver T, 2014. Transcriptomewide identification of bread wheat WRKY transcription factors in response to drought stress. Mol. Genet. Genomic. 289(5): 765-781.
Qin Y, Tian Y and Liu X, 2015. A wheat salinityinduced WRKY transcription factor TaWRKY93 confers multiple abiotic stress tolerance in Arabidopsis thaliana. Biochem. Biophys. Res. Commun. 464(2): 428-433.

Rehman SU, Bilal M, Rana RM, Tahir MN, Shah MK, Ayalew H and Yan G, 2016. Cell membrane stability and chlorophyll content variation in wheat (Triticum aestivum) genotypes under conditions of heat and drought. Crop Pasture Sci. 67(7): 712-718.

Rushton PJ, Somssich IE, Ringler P and Shen QJ, 2010. WRKY transcription factors. Trends Plant Sci. 15(5): 247-258.

Sibley JL, Eakes DJ, Gilliam CH, Keever GJ, Dozier WA and Himelrick DG, 1996. Foliar SPAD-502 meter values, nitrogen levels, and extractable chlorophyll for red maple selections. HortSci. 31(3): 468-470.

Simova-Stoilova L, Demirevska K, Petrova T, Tsenov $\mathrm{N}$ and Feller U, 2008. Antioxidative protection in wheat varieties under severe recoverable drought at seedling stage. Plant Soil Environ. 54(12): 529536.

Wang C, Deng P, Chen L, Wang X, Ma H, Hu W, Yao N, Feng Y, Chai R, Yang G and He G, 2013. A wheat WRKY transcription factor TaWRKY10 confers tolerance to multiple abiotic stresses in transgenic tobacco. PloS One. 8(6): e65120.

$\mathrm{Xu}$ ZS, Chen M, Li LC and Ma YZ, 2011. Functions and application of the AP2/ERF transcription factor family in crop improvement. J. Integr. Plant Biol. 53(7): 570-585.

Zhou S, Zheng WJ, Liu BH, Zheng JC, Dong FS, Liu ZF, Wen ZY, Yang F, Wang HB, Xu ZS and Zhao H, 2019. Characterizing the Role of TaWRKY13 in salt Tolerance. Int. J. Mol. Sci. 20(22): 5712.

\section{Contribution of Authors}

Darwish E: Conceived idea, designed study, performed the experiment and wrote the manuscript

Rehman SU: Collected and analyzed data and wrote the manuscript

Mao X \& Jing R: Supervised research, edited manuscript and gave final approval 